J. S. PAK

KODAI MATH. J.

1 (1978), 187-196

\title{
PLANAR GEODESIC SUBMANIFOLDS IN COMPLEX SPACE FORMS
}

\author{
By JIN SUK PAK
}

Let $M^{n}$ and $\bar{M}^{n+p}$ be connected complete Riemannian manifolds of dimension $n$ and $n+p$ respectively. An isometric immersion of $M^{n}$ into $\bar{M}^{n+p}$ is called a planar geodesic immersion when every geodesic in $M^{n}$ is mapped locally into 2-dimensional totally geodesic submanifold of $\bar{M}^{n+p}$. When the ambient manifold $\bar{M}^{n+p}$ is a space form of constant curvature $\tilde{c}$, K. Sakamoto [7] has showed that such an immersion is an isotropic immersion in the sence of B. O'Neill [6] with parallel second fundamental tensor. Using this fact, he reduced planar geodesic immersions into space forms to full, minimal and planar geodesic immersions of compact rank one symmetric spaces into spheres and obtained

THEOREM A. Let $f: M^{n} \longrightarrow S^{n+q}(\tilde{c})$ be a planar geodesic immersion. Then the simply connected Riemannian covering manifold of $M^{n}$ is a sphere, a complex projective space, a quaternionic projective space or a Cayley projective plane. The immersion is rigid.

A submanifold $M^{n}$ of a complex space form $\bar{M}^{n+p}(\tilde{c})$ with constant holomorphic sectional curvature $\tilde{c}$ is called complex or invariant (resp. totolly real) if each tangent space of $M^{n}$ is mapped into itself (resp. the normal space) by the complex structure of $\bar{M}^{n+p}(\tilde{c})$. A complex submanifold of a Kaehler manifold is also a Kaehler manifold. K. Ogiue [5] has showed that if $M^{n}(c)$ is a Kaehler submanifold immersed in $\bar{M}^{n+p}(\tilde{c})$ and if the second fundamental form of the immersion is parallel, then either $c=\tilde{c}$ (i. e., $M^{n}(c)$ is totally geodesic in $\bar{M}^{n+p}(\tilde{c})$ ) or $c=\tilde{c} / 2$, the latter case arising only when $\tilde{c}>0$. Moreover the immersion is rigid. When $\tilde{c} \leqq 0$, E. Calabi [1] proved that if $M^{n}(c)$ is imbedded in $\bar{M}^{n+p}(\tilde{c})$ as a Kaehler submanifold, then $M^{n}(c)$ is totally geodesic in $\bar{M}^{n+p}(\tilde{c})$.

In this paper we study a planar geodesic immersion $f: M^{n} \longrightarrow \bar{M}^{n+p}(\tilde{c})$ of a connected complete Riemannian manifold of real dimension $n$ into a complex space form of real dimension $n+p$ with constant holomorphic sectional curvature $\tilde{c} \neq 0$. When the immersion $f$ is complex or totally real, it is an isotropic immersion with parallel second fundamental tensor. Moreover, if the immersion $f$ is totally real and not totally geodesic, we can reduce the immersion to a full, minimal, planar geodesic immersion of $M^{n}$ into a real projective space $R P^{n+p}(\tilde{c} / 4)$ (resp. a real hyperbolic space $H^{n+p}(\tilde{c} / 4)$ ) when $\bar{M}^{n+p}(\tilde{c})$ is a complex projective

Received September 24, 1976. 
space $C P^{m}(\tilde{c})$ (resp. a complex hyperbolic space $D^{m}(\tilde{c})$ ), where $m=n+p / 2$. Roughly speaking, our results are due to Calabi-Ogiue's results in the case where $M^{n}$ is complex and due to Theorem A in the case where $M^{n}$ is totally real.

Manifolds, tensor fields, geometric objects and mappings we consider are assumed to be differentiable and of class $C^{\infty}$.

The author would like to express his hearty thanks to Professor Kunio Sakamoto for his valuable suggestions and encouragements.

\section{$\S 1$. Preliminaries.}

Let $M^{n}$ and $\bar{M}^{n+p}$ be connected complete Riemannian manifolds with real dimension $n(\geqq 2)$ and $n+p$ respectively and let $f: M^{n} \longrightarrow \bar{M}^{n+p}$ an isometric immersion. We denote by $\bar{\nabla}$ the covariant differentiation with respect to the Riemannian metric of $\bar{M}^{n+p}$. Then we may write

$$
\bar{\nabla}_{X} Y=\nabla_{X} Y+H(X, Y)
$$

for arbitrary tangent vector fields $X$ and $Y$ on $M$, where $\nabla_{X} Y$ and $H(X, Y)$ denote the components of $\bar{\nabla}_{X} Y$ tangent and normal to $M^{n}$ respectively. Then $\nabla$ becomes the covariant differentiation of the Riemannian manifold $M^{n}$. The symmetric bilinear form $H$ valued in the normal space is called the second fundamental form of the immersion $f$. For a normal vector field $C$ on a neighborhood of $P \in M^{n}$, we write

$$
\bar{\nabla}_{X} C=-A_{C} X+\nabla_{X}^{\frac{1}{X}} C,
$$

$-A_{C} X$ and $\nabla_{X}^{\perp} C$ being the components of $\bar{\nabla}_{X} C$ tangent and normal to $M^{n}$ respectively, where $\nabla^{\perp}$ is the covariant differentiation with respect to the induced connection in the normal bundle $N M$ which will be called the normal connection. Denoting the inner product of vectors with respect to the Riemannian metric of $\bar{M}^{n+p}$ by $\langle$,$\rangle , we find that the tangential component -A_{C} X$ of $\bar{\nabla}_{X} C$ and the second fundamental form $H$ are related by

$$
\left\langle A_{C} X, Y\right\rangle=\langle H(X, Y), C\rangle
$$

for any vector $Y$ contained in the tangent space $T_{P} M^{n}$. Thus $A_{C}$ is a symmetric linear transformation of $T_{P} M^{n}$. Given an orthonormal normal frame $\left\{C_{n+1}, \cdots, C_{n+p}\right\}$, we write $A_{\alpha}=A_{C_{\alpha}}(\alpha=n+1, \cdots, n+p)$. In the sequel, indices $\alpha$, $\beta$ and $\gamma$ run over the range $\{n+1, \cdots, n+p\}$.

Let $' \nabla$ be the covariant differentiation with respect to the induced connection in the direct sum (tangent bundle $T M) \oplus$ (normal bundle $N M$ ). For the second fundamental form $H$, we find

$$
\left({ }^{\prime} \nabla_{X} H\right)(Y, Z)=\nabla_{X}^{\perp}(H(Y, Z))-H\left(\nabla_{X} Y, Z\right)-H\left(Y, \nabla_{X} Z\right)
$$

for all tangent vector fields $X, Y$ and $Z$ on $M$. 
Let the ambient manifold $\bar{M}^{n+p}$ be a complete, simply connected complex space form of real dimension $n+p$ with constant holomorphic sectional curvature $\tilde{c} \neq 0$. The complex dimension will be denoted by $m=(n+p) / 2$. Thus $\bar{M}^{n+p}$ will be complex projective space $C P^{m}(\tilde{c})$ or complex hyperbolic space $D^{m}(\tilde{c})$ according as $\tilde{c}>0$ or $\tilde{c}<0$. If $\bar{J}$ denotes the complex structure, the Riemannian curvature tensor of the complex space form $\bar{M}^{m}(\tilde{c})$ is given by

$$
\begin{aligned}
\bar{R}(\bar{X}, \bar{Y}) \bar{Z}=(\tilde{c} / 4)(\langle\bar{Y}, \bar{Z}\rangle \bar{X} & -\langle\bar{X}, \bar{Z}\rangle Y+\langle\bar{J} \bar{Y}, \bar{Z}\rangle \bar{J} \bar{X} \\
& -\langle\bar{J} \bar{X}, \bar{Z}\rangle \bar{J} \bar{Y}-2\langle\bar{J} \bar{X}, \bar{Y}\rangle \bar{J} \bar{Z})
\end{aligned}
$$

for all $\bar{X}, \bar{Y}, \bar{Z}$ tangent to $\bar{M}^{m}(\tilde{c})$.

We denote by $\operatorname{Proj}_{T M}$ and $\operatorname{Proj}_{N M}$ the projections of $T_{P} \bar{M}^{m}(\tilde{c})$ to the tangent space $T_{P} M^{n}$ and the normal space $N_{P} M^{n}$ respectively and put $J=\operatorname{Proj}_{T M} \circ \bar{J} \mid T M$, $J_{N}=\operatorname{Proj}_{N M} \circ \bar{J}\left|T M, J_{T}=\operatorname{Proj}_{T M} \circ \bar{J}\right| N M$ and $J^{\perp}=\operatorname{Proj}_{N M^{\circ}} \bar{J} \mid N M$. Then we can write

$$
\bar{J} X=J \bar{X}+J_{N} X, \quad \bar{J} C=J_{T} C+J^{\perp} C
$$

for all $X$ tangent to $M^{n}$. Taking account of $\bar{J}^{2}=-I$, we see that these tensors satisfy

$$
\begin{aligned}
& J^{2}+J_{T} J_{N}=-I, \quad J_{N} J+J^{\perp} J_{N}=0, \\
& J^{\perp 2}+J_{N} J_{T}=-I, \quad J J_{T}+J_{T} J^{\perp}=0,
\end{aligned}
$$

$I$ denoting the identity transformation, and also we find

$$
\left\langle J_{N} X, C\right\rangle=-\left\langle X, J_{T} C\right\rangle
$$

with the help of $\langle\bar{J} X, Y\rangle=-\langle X, \bar{J} Y\rangle$.

Differentiating covariantly the left hand side of (1.6), we have

$$
\bar{\nabla}_{Y} \bar{J} X=\left(\nabla_{Y} J\right) X+J\left(\nabla_{Y} X\right)-A_{J_{N} X} Y+\left({ }^{\prime} \nabla_{Y} J_{N}\right) X+J_{N}\left(\nabla_{Y} X\right)+H(J X, Y)
$$

because of (1.1) and (1.2). On the other hand, using $\bar{\nabla} \bar{J}=0$ and (1.6) itself, we also have

$$
\bar{\nabla}_{Y} \bar{J} X=J\left(\nabla_{Y} X\right)+J_{T} H(X, Y)+J_{N}\left(\nabla_{Y} X\right)+J^{\perp} H(X, Y),
$$

from which

$$
\begin{aligned}
\left(\nabla_{Y} J\right) X & =A_{J_{N} X} Y+J_{T} H(X, Y), \\
\left(\nabla_{Y} J_{N}\right) X & =J^{\perp} H(X, Y)-H(J X, Y) .
\end{aligned}
$$

Similarly, from the right hand side of (1.6), we also obtain

$$
\begin{gathered}
\left({ }^{\prime} \nabla_{X} J_{T}\right) C=A_{J^{\perp} C} X-J A_{C} X, \\
\left({ }^{\prime} \nabla_{X} J^{\perp}\right) C=-J_{N} A_{C} X-H\left(X, J_{T} C\right) .
\end{gathered}
$$


Let's denote curvature tensors for the connections $\nabla$ and $\nabla^{\perp}$ by $R$ and $R^{\perp}$ respectively. Then, using (1.5), we can easily see that the structure equations of Gauss are given by

$$
\begin{aligned}
R(X, Y) Z= & (\tilde{c} / 4)(\langle Y, Z\rangle X-\langle X, Z\rangle Y+\langle J Y, Z\rangle J X \\
& -\langle J X, Z\rangle J Y-2\langle J X, Y\rangle J Z) \\
& +\sum_{\alpha}\left(\left\langle A_{\alpha} Y, Z\right\rangle A_{\alpha} X-\left\langle A_{\alpha} X, Z\right\rangle A_{\alpha} Y\right),
\end{aligned}
$$

or equivalently

$$
\begin{aligned}
\langle R(X, Y) Z, W\rangle= & (\tilde{c} / 4)(\langle Y, Z\rangle\langle X, W\rangle-\langle X, Z\rangle\langle Y, W\rangle \\
& +\langle J Y, Z\rangle\langle J X, W\rangle-\langle J X, Z\rangle\langle J Y, W\rangle \\
& -2\langle J X, Y\rangle\langle J Z, W\rangle) \\
& +\langle H(Y, Z), H(X, W)\rangle-\langle H(X, Z), H(Y, W)\rangle,
\end{aligned}
$$

and those of Coddazi and Ricci respectively by

$$
\begin{gathered}
\left({ }^{\prime} \nabla_{X} H\right)(Y, Z)-\left({ }^{\prime} \nabla_{Y} H\right)(X, Z) \\
=(\tilde{c} / 4)\left(\langle J Y, Z\rangle J_{N} X-\langle J X, Z\rangle J_{N} Y\right. \\
\left.\quad-2\langle J X, Y\rangle J_{N} Z\right), \\
R^{\perp}(X, Y) C=(\tilde{c} / 4)\left(\left\langle J_{N} Y, C\right\rangle J_{N} X-\left\langle J_{N} X, C\right\rangle J_{N} Y\right. \\
\left.-2\langle J X, Y\rangle J^{\perp} C\right)+\sum_{\alpha}\left\langle\left[A_{C}, A_{\alpha}\right] X, Y\right\rangle C_{\alpha} .
\end{gathered}
$$

Therefore, if the submanifold $M^{n}$ is complex or totally real, that is, $J_{N}=0$ or $J=0$, then

$$
\left({ }^{\prime} \nabla_{X} H\right)(Y, Z)-\left({ }^{\prime} \nabla_{Y} H\right)(X, Z)=0
$$

with the help of (1.12). Conversely if the above equation is verified at every point of $M^{n}$, then $M^{n}$ is complex or totally real. Hence 2-dimensional complete totally geodesic submanifolds in $C P^{m}(\tilde{c})$ are $C P^{1}(\tilde{c})$ or $R P^{2}(\tilde{c} / 4)$.

\section{§2. Planar geodesic submanifolds.}

We consider an isometric immersion $f: M^{n} \longrightarrow \bar{M}^{m}(\tilde{c})$ such that every geodesic $\sigma:(a, b) \longrightarrow M^{n}$ on $M^{n}$ is locally contained in a 2-dimensional totally geodesic submanifold of $\bar{M}^{m}(\tilde{c})$, that is, for each $t \in(a, b)$, there exist an open interval $I, t \in I \subset(a, b)$ and 2-dimensional totally geodesic submanifold $M^{2}{ }_{t}$ such that $f(\sigma(I)) \subset M^{2}{ }_{t}$. In this case the immersion $f$ is called a planar geodesic immersion and the manifold $M^{n}$ called a planar geodesic submanifold. Then we have 
Lemma 2.1. (S. L. Hong [3] and K. Sakamoto [7]). Let $X$ and $Y$ are orthonormal vectors at $P \in M^{n}$ in TM. Then

$$
\langle H(X, X), H(X, Y)\rangle=0 .
$$

The equation (2.1) is equivalent to the condition that $f$ is isotropic, i. e.,

$$
\|H(X, X)\|^{2}=\lambda^{2}
$$

for all unit vector $X$ tangent to $M^{n}$, where $\lambda$ is a differentiable function on $M^{n}$. In fact, in this case the function $\lambda^{2}$ is given by

$$
\lambda^{2}=1 / n(n+2) \sum_{\alpha}\left\{\left(\operatorname{trace} A_{\alpha}\right)^{2}+\operatorname{trace} A_{\alpha}{ }^{2}\right\}
$$

(See K. Sakamoto [7]). We first prove

LEMMA 2.2. The function $\lambda^{2}$ is constant on $M^{n}$.

Proof. Let $P$ be arbitrary fixed point of $M^{n}$. Take normal coordinate neighborhood $U$ around $P$ in $M^{n}$. Let $X$ be any unit vector tangent to $M^{n}$ at $P$ and $Y$ an unit vector orthogonal to $X$ at $P$. Let $\sigma$ be a geodesic with unit speed such that $\sigma(0)=P$ and $\dot{\sigma}(0)=Y$. Then, using (1.1), we find

$$
\bar{\nabla}_{f \dot{\sigma}} f \dot{\sigma}=f \nabla_{\dot{\sigma}} \dot{\sigma}+H(\dot{\sigma}, \dot{\sigma})=H(\dot{\sigma}, \dot{\sigma}) .
$$

We assume now $H(Y, Y)=H(\dot{\sigma}(0), \dot{\sigma}(0)) \neq 0$. Then, since $M^{2}$ is totally geodesic submanifold in $\bar{M}^{m}(\tilde{c})$, we have $\bar{\nabla}_{f \dot{\sigma}} H(\dot{\sigma}, \dot{\sigma}) \in T_{f \cdot \sigma} M^{2}$ and hence

$$
\bar{\nabla}_{f \dot{\sigma}} H(\dot{\sigma}, \dot{\sigma})=a(t) f \dot{\sigma}+b(t) H(\dot{\sigma}, \dot{\sigma})
$$

for some differentiable functions $a(t)$ and $b(t)$. Translate $X$ and $Y$ parallely to each point of $U$ along the unique geodesic from $P$ to that point. Then we have vector fields defined on $U$ denoted by $\bar{X}$ and $\bar{Y}$ which extend $X$ and $Y$ respectively and satisfy

$$
\nabla_{X} \bar{X}=\nabla_{Y} \bar{Y}=\nabla_{X} \bar{Y}=\nabla_{Y} \bar{X}=0 \quad \text { at } P .
$$

We can compute $\left(X \cdot \lambda^{2}\right)(P)$ as followings :

$$
\begin{aligned}
\left(X \cdot \lambda^{2}\right)(P)= & X \cdot\langle H(\bar{Y}, \bar{Y}), H(\bar{Y}, \bar{Y})\rangle\left|P=2\left\langle\overline{\nabla_{\bar{X}}}(H(\bar{Y}, \bar{Y})), H(\bar{Y}, \bar{Y})\right\rangle\right| P \\
= & 2\left\langle\nabla_{\bar{X}}(H(\bar{Y}, \bar{Y})), H(\bar{Y}, \bar{Y})\right\rangle \mid P \\
= & 2\left\langle\left(^{\prime} \nabla_{\bar{X}} H\right)(\bar{Y}, \bar{Y})+2 H\left(\nabla_{\bar{X}} \bar{Y}, \bar{Y}\right), H(\bar{Y}, \bar{Y})\right\rangle \mid P \\
= & 2\left\langle\left(^{\prime} \nabla_{\bar{X}} H\right)(\bar{Y}, \bar{Y}), H(\bar{Y}, \bar{Y})\right\rangle \mid P \\
= & 2\left\langle\left({ }^{\prime} \nabla_{\bar{Y}} H\right)(\bar{X}, \bar{Y})+(\tilde{c} / 4)\left(\langle J \bar{Y}, \bar{Y}\rangle J_{N} \bar{X}-\langle J \bar{X}, \bar{Y}\rangle J_{N} \bar{Y}\right.\right. \\
& \left.\left.\quad-2\langle J \bar{X}, \bar{Y}\rangle J_{N} \bar{Y}\right), H(Y, Y)\right\rangle \mid P
\end{aligned}
$$




$$
\begin{aligned}
= & 2\left\langle\nabla_{\bar{Y}}^{\frac{1}{Y}}(H(\bar{X}, \bar{Y})), H(\bar{Y}, \bar{Y})\right\rangle \mid P \\
& -(3 / 2) \tilde{c}\langle J X, Y\rangle\left\langle J_{N} Y, H(Y, Y)\right\rangle \\
= & 2\left\langle\bar{\nabla}_{\bar{Y}}(H(\bar{X}, \bar{Y})), H(\bar{Y}, \bar{Y})\right\rangle \mid P \\
& -(3 / 2) \tilde{c}\langle J X, Y\rangle\left\langle J_{N} Y, H(Y, Y)\right\rangle \\
= & -2\left\langle H(\bar{X}, \bar{Y}), \bar{\nabla}_{\bar{Y}}(H(\bar{Y}, \bar{Y}))\right\rangle \mid P \\
& -(3 / 2) \tilde{c}\langle J X, Y\rangle\left\langle J_{N} Y, H(Y, Y)\right\rangle
\end{aligned}
$$

with the help of (1.12), (2.1) and (2.2). Since $\bar{\nabla}_{\bar{Y}}(H(\bar{Y}, \bar{Y}))\left|P=\bar{\nabla}_{f \dot{\sigma}}(H(\dot{\sigma}, \dot{\sigma}))\right|_{t=0}$ $=a(0) f Y+b(0) H(Y, Y)$, the first term of the last equation vanishes. Hence we obtain

$$
\left(X \cdot \lambda^{2}\right)(P)=(-3 / 2) \tilde{c}\langle J X, Y\rangle\left\langle J_{N} Y, H(Y, Y)\right\rangle .
$$

Since $P$ is taken arbitrary in $M^{n}$, for orthonormal vectors $X$ and $Y$

$$
\left(X \cdot \lambda^{2}\right)=(-3 / 2) \tilde{c}\langle\bar{J} X, Y\rangle\langle\bar{J} Y, H(Y, Y)\rangle .
$$

If $M^{2}$ is invariant, then $\bar{J} Y \in T_{P} M^{2}$ and hence $H(Y, Y)= \pm \lambda \bar{J} Y$. Thus we have $X \cdot \lambda^{2}=0$. If $M^{2}$ is totally real, then $\bar{J} Y$ is orthogonal to $T_{P} M^{2}$ and consequently $\bar{J} Y$ is orthogonal to $H(Y, Y)$. Thus we have $X \cdot \lambda^{2}=0$. Therefore we have proved $\left(X \cdot \lambda^{2}\right)(P)=0$ for arbitrary $X$ if $\lambda^{2}(P) \neq 0$. When $\lambda^{2}(P)=0, \quad \lambda^{2}$ takes minimum at $P$. Thus $\left(X \cdot \lambda^{2}\right)(P)=0$ for any $X$. These complete the proof.

Q.E.D.

From the above proof we have

$$
H(X, X)= \pm \lambda \bar{J} X \quad \text { or } H(X, X) \perp \bar{J} X
$$

for each unit vector $X$ tangent to $M^{n}$. But if we regard $\langle H(X, X), \bar{J} X\rangle$ as a function on the unit sphere bundle, then this function is clearly differentiable (continuous). Hence the above relations are established for all unit vectors $X$. Thus we have

LEMMA 2.3. Every geodesic is contained in a totally geodesic submanifold $C P^{1}(\tilde{c})$ or $R P^{2}(\tilde{c} / 4)$.

In the sequel, we denote by $(\mathrm{C})$ the case where $H(X, X)=\lambda \bar{J} X$ holds on the unit sphere bundle and by $(\mathrm{R})$ the case where $H(X, X) \perp \bar{J} X$ always holds on the unit sphere bundle.

Finally we prepare the following lemma.

Lemma 2.4. For all vectors $X, Y$ and $Z$ tangent to $M^{n}$

$$
\left(\nabla_{X} H\right)(Y, Z)=(-\tilde{c} / 4)\left(\langle J X, Y\rangle J_{N} Z+\langle J X, Z\rangle J_{N} Y\right) .
$$


Proof. Let $X$ be any unit vector at any point $P \in M^{n}$. Let $\sigma$ be a unit speed geodesic such that $\sigma(0)=P$ and $\dot{\sigma}(0)=X$. We may assume that $\lambda \neq 0$. Since $\|H(\dot{\sigma}, \dot{\sigma})\|^{2}=\lambda^{2}=$ constant, and $\lambda \neq 0$, we can see that $\bar{\nabla}_{f \dot{\sigma}}(H(\dot{\sigma}, \dot{\sigma}))=-\lambda^{2} f \dot{\sigma}$. On the other hand, we also have

$$
\bar{\nabla}_{f \dot{\sigma}}(H(\dot{\sigma}, \dot{\sigma}))=-f A_{H(\dot{\sigma}, \dot{\sigma})} \dot{\sigma}+\nabla_{\dot{\sigma}}^{\dot{1}}(H(\dot{\sigma}, \dot{\sigma})) .
$$

Thus $\nabla_{\dot{\sigma}}^{\frac{1}{\sigma}}(H(\dot{\sigma}, \dot{\sigma}))=0$ and $A_{H(\dot{\sigma}, \dot{\sigma})} \dot{\sigma}=\lambda^{2} \dot{\sigma}$. These imply

$$
(' \nabla H)(X, X, X)=0 \text { and } A_{H(X, X)} X=\lambda^{2} X\langle X, X\rangle
$$

for any vector $X$. Therefore we obtain

$$
\Im(' \nabla H)(X, Y, Z)=0
$$

and

$$
\Im \sum_{\alpha}\left\langle A_{\alpha} X, Y\right\rangle A_{\alpha} Z=\lambda^{2} \varsigma\langle X, Y\rangle Z,
$$

where $\subseteq$ denotes the cyclic sum with respect to $X, Y$ and $Z$. The latter is the isotropic condition. The former reduces to

$$
\left({ }^{\prime} \nabla_{X} H\right)(Y, Z)+\left({ }^{\prime} \nabla_{Y} H\right)(Z, X)+\left({ }^{\prime} \nabla_{Z} H\right)(X, Y)=0,
$$

from which, taking account of (1.12), we have

$$
\begin{aligned}
0= & \left({ }^{\prime} \nabla_{X} H\right)(Y, Z)+\left({ }^{\prime} \nabla_{X} H\right)(Y, Z)+(\tilde{c} / 4)\left(\langle J X, Z\rangle J_{N} Y\right. \\
& \left.-\langle J Y, Z\rangle J_{N} X-2\langle J Y, X\rangle J_{N} Z\right)+\left({ }^{\prime} \nabla_{X} H\right)(Y, Z) \\
& +(\tilde{c} / 4)\left(\langle J X, Y\rangle J_{N} Z-\langle J Z, Y\rangle J_{N} X-2\langle J Z, X\rangle J_{N} Y\right) \\
= & 3\left({ }^{\prime} \nabla_{X} H\right)(Y, Z)+(3 \tilde{c} / 4)\left(\langle J X, Y\rangle J_{N} Z+\langle J X, Z\rangle J_{N} Y\right),
\end{aligned}
$$

and hence (2.3).

Q.E.D.

Therefore we see that if the submanifold $M^{n}$ is complex or totally real, then the second fundamental form is parallel.

\section{§ 3. The case $(\mathrm{C})$.}

In $\S \S 3$ and 4 we shall study only planar geodesic submanifolds of complex projective space $C P^{m}(\tilde{c})$ and those of complex hyperbolic space $D^{m}(\tilde{c})$.

First we consider the case (C), that is, the case where $H(X, X)=\lambda \bar{J} X$ holds on the unit sphere bundle. In this case $\lambda=0$ and consequently we have

Proposition 1. The case (C) occurs only if $M^{n}$ is totally geodesic, $M^{n}=$ $R P^{n}(\tilde{c} / 4)$ or $C P^{n / 2}(\tilde{c})$ and the immersion is rigid. 


\section{$\S 4$. The case $(\mathrm{R})$.}

Next, we consider the case (R), that is, the case that $H(X, X) \perp \bar{J} X$ holds on the unit sphere bundle. Then we have $\left\langle H(X, X), J_{N} X\right\rangle=0$ for any vector $X$. Therefore, symmetrizing this equation, we have

LEMMA 4.1. In the case (R) the following equations hold:

$$
\Im\left\langle H(X, Y), J_{N} Z\right\rangle=0 \text {. }
$$

We first assume that the planar geodesic submanifold $M^{n}$ is a Kaehler submanifold. Then the second fundamental form is parallel because of Lemma 2.4. On the other hand, since the immersion $f$ is isotropic, $M^{n}$ is of constant holomorphic sectional curvature $\tilde{c}-2 \lambda^{2}$. In fact, using $(1.11)^{\prime}$, we find

$$
\langle R(X, J X) J X, X\rangle=\tilde{c}-2\|H(X, X)\|^{2}=\tilde{c}-2 \lambda^{2}
$$

for arbitrary unit vector $X$. Since the second fundamental form is parallel, we can also see that $\tilde{c}-2 \lambda^{2}=\tilde{c} / 2$ (i. e., $\lambda^{2}=\tilde{c} / 4$ ). This conclusion is due to Ogiue [5]. He obtained his results by establishing an equation of Simon's type. Thus we have

Proposition 2. If a planar geodesic submanifold $M^{n}$ of $C P^{m}(\tilde{c})$ is a Kaehler submanifold, then it is $C P^{n / 2}(\tilde{c} / 2)$ or $C P^{n / 2}(\tilde{c})$, i.e., a complex Veronese manifold or totally geodesic. The immersion is rigid.

We next consider the case where the planar geodesic submanifold $M^{n}$ is totally real. We then have $A_{J_{N} X} Y+J_{T} H(X, Y)=0$ which is a direct consequence of $J=0$ and (1.9). Therefore $\left\langle A_{J_{N} X} Y, Z\right\rangle=-\left\langle J_{T} H(X, Y), Z\right\rangle$ and consequently $\left\langle J_{N} X, H(Y, Z)\right\rangle=-\left\langle X, J_{T} H(Y, Z)\right\rangle=\left\langle A_{J_{N} Z} Y, X\right\rangle=\left\langle J_{N} Z, H(X, Y)\right\rangle$. Thus we find $\left\langle J_{N} X, H(Y, Z)\right\rangle=\left\langle J_{N} Z, H(X, Y)\right\rangle=\left\langle J_{N} Y, H(Z, X)\right\rangle$, from which and Lemma 4.1,

$$
\left\langle J_{N} X, H(Y, Z)\right\rangle=0
$$

Moreover the second fundamental form is parallel too. Using these facts, we shall reduce the codimension to the dimension of the first normal space. The first normal space $N_{1}(P)$ at $P$ is defined as the subspace in the normal space $N_{P} M^{n}$ which is spanned by the set $\left\{H(X, Y): X, Y \in T_{P} M^{n}\right\}$. Denoting by $S p\{\}$ the vector space spanned by the set \{\} of vectors, we can see (cf. Sakamoto [7])

$$
N_{1}(P)=S p\left\{C: A_{C}=0\right\}_{P}^{\frac{1}{P}}
$$

and

$$
N_{1}(P) \approx S p\left\{A_{C}: C \in N_{P} M^{n}\right\}
$$


in the vector space consisting of symmetric linear transformations on $T_{P} M^{n}$, where ${ }^{\perp}$ means the orthogonal complement in $N_{P} M^{n}$.

LEMMA 4.1. (Sakamoto [7]). If a planar geodesic submanifold $M^{n}$ of $C P^{m}(\tilde{c})$ is totally real and of type $(\mathrm{R})$, then the first normal space $N_{1}$ is parallel with respect to the normal connection and is orthogonal to $J_{N}(T M)$ in the normal bundle, i. e., $N_{1} \perp J_{N}(T M)$.

Proof. Let $P$ and $Q$ be arbitrary two points of $M^{n}$ and $\sigma$ a curve from $P$ to $Q$ in $M^{n}$. Let $\left\{X_{1}, \cdots, X_{n}\right\}$ be an orthonormal base of $T_{P} M^{n}$. Then $N_{1}(P)=$ $S p\left\{H\left(X_{2}, X_{j}\right): i, \jmath=1,2, \cdots, n\right\}$. Translate parallely this orthonormal base from $P$ to $Q$ along $\sigma$ with respect to the Riemannian connection of $M^{n}$. Then we have orthonormal frame field parallel along $\sigma$, which will be also denoted by $\left\{X_{1}, \cdots, X_{n}\right\}$. Thus $H\left(X_{\imath}, X_{j}\right)$ is parallel along $\sigma$ with respect to the induced connection in the normal bundle for each $\imath$ and $\jmath$, because

$$
\nabla_{\sigma}^{\perp} H\left(X_{\imath}, X_{j}\right)=\left({ }^{\prime} \nabla_{\dot{\sigma}} H\right)\left(X_{\imath}, X_{\jmath}\right)+H\left(\nabla_{\sigma} X_{\imath}, X_{\jmath}\right)+H\left(X_{\imath}, \nabla_{\dot{\sigma}} X_{\jmath}\right)=0,
$$

where we have used $\rightarrow \nabla H=0$. It follows that the parallel displacement along $\sigma$ from $P$ to $Q$ with respect to the induced connection in the normal bundle gives an isomorphism of $N_{1}(P)$ to $N_{1}(Q)$. Hence the dimension of $N_{1}$ is constant and $N_{1}$ is invariant by the parallel displacement with respect to $\nabla^{\perp}$ and hence $N_{1} \perp J_{N}(T M)$ with the help of (4.1).

Q.E.D.

Since $\left\langle J_{N} X, H(Y, Z)\right\rangle=0$, we see that $H(Y, Z)=0$, i. e., that $M^{n}$ is totally geodesic, when $p=n$. Thus we have

Proposition 3. If a planar geodesıc submanifold $M^{n}$ of $C P^{n}(\tilde{c})$ is totally real and type (R), then $M^{n}$ is totally geodesic.

Now, we suppose $p>n$. Then we have

LEMMA 4.2. The subspace $N_{1} \oplus T M$ is totally real.

Proof. From $\left\langle J_{N} X, H(Y, Z)\right\rangle=0$ we can easily see that $J_{T} N_{1}=0$. Differentiating covariantly the equation $\left\langle J_{N} X, H(Y, Z)\right\rangle=0$ with respect to ${ }^{\prime} \nabla$ and using ${ }^{\prime} \nabla H=0$ and (1.9), we have $J^{\perp} N_{1} \perp N_{1}$ and so the assertion is followed from Lemma 4. 1.

Q. E. D.

LEMMA 4.3. There exists a totally geodesic and totally real submanifold $R P^{n+q}(\tilde{c} / 4)$ with constant sectronal curvature $\tilde{c} / 4$ such that $f\left(M^{n}\right) \subset R P^{n+q}(\tilde{c} / 4)$ and the immersion $f: M^{n} \longrightarrow R P^{n+q}(\tilde{c} / 4)$ is full, where $q=\operatorname{dim} N_{1}$.

Proof. From Lemma 4.2 it follows that there exists a unique totally geodesic and totally real submanifold $R P^{n+q}(\tilde{c} / 4)$ tangent to $N_{1}(P) \oplus T_{P} M^{n}$ at distinguished point $P \in M^{n}$. Let $\sigma$ be a geodesic starting at $P$ and ending at an arbitrary point $Q \in M^{n}$. Then $f \cdot \sigma$ is contained in $R P^{2}$ spanned by $f \dot{\sigma}(0)$ and 
$H(\dot{\sigma}(0), \dot{\sigma}(0))$. Thus $f \cdot \sigma \subset R P^{2} \subset R P^{n+q}(\tilde{c} / 4)$. Clearly, the immersion $f: M^{n}$ $\longrightarrow R P^{n+q}(\tilde{c} / 4)$ is full.

Q.E.D.

Therefore we can reduce our immersion to a full, planar geodesic immersion $f: M^{n} \longrightarrow R P^{n+q}(\tilde{c} / 4)$. We may assume locally that the immersion $f: M^{n} \longrightarrow$ $S^{n+q}(\tilde{c} / 4)$ is planar and full. Thus, combining Theorem A, we have

PROPOSITION 4. If a planar geodesic submanifold $M^{n}$ of $C P^{m}(\tilde{c})$ is totally real and of type (R), then $M^{n}$ is a compact irreducible symmetruc space of rank one, i.e., $M^{n}$ is one of a sphere, a real projective space, a complex projective space, a quaternonic projective space and a Cayley projectıve plane.

Remark. Let the ambient manifold $\bar{M}^{m}(\tilde{c})(m=(n+p) / 2)$ is complex hyperbolic space $D^{m}(\tilde{c})$. When the case $(C)$ is arised, we can also see that a planar geodesic submanifold $M^{n}$ of $D^{m}(\tilde{c})$ is totally geodesic by means of Proposition 1. When the submanifold $M^{n}$ is complex and of type (R), $M^{n}$ is totally geodesic in $D^{m}(\tilde{c})$ because it is also of constant holomorphic sectional curvature. Finally when the immersion $f: M^{n} \longrightarrow D^{m}(\tilde{c})$ is totally real and of type (R), we can also reduce the immersion to a full, planar geodesic immersion $f: M^{n} \longrightarrow$ $H^{n+q}(\tilde{c} / 4)$. Hence, using Theorem A, we find that $M^{n}$ is a sphere, a complex projective space, a quaternionic projective space or a Cayley projective plane and the immersion is rigid.

\section{BIBLIOGRAPHY}

[1] E. CALABI, Isometric imbedding of complex manifolds, Ann. of Math., 58 (1953), 1-23.

[2] B. Y. CHen and K. OGIUe, On totally real submanifolds, Transactions of AMS., 193 (1974), 257-266.

[3] S.L. Hong, Isometric immersions of manifolds with plane geodesics into Euclidean space, J. Diff. Geometry, 8 (1973), 259-278.

[4] K. Nomizu, Eine Kennzeichnung der projektıven Unterräume in dem komplexen projektiven Raum nach ihren Geodätischen, Math. Z., 137 (1974), 147-150.

[5] K. Ogiue, On Kaehler immersions, Can. J. Math., 24 (1972), 1178-1182.

[6] B. O'Neill, Isotropic and Kaehler immersions, Can. J. Math., 17 (1965), 907915.

[7] K. Sakamoto, Planar geodesic immersions, to appear.

KYUNGPOOK UNIVERSITY

TAE-GU, KOREA 